\title{
Nonoperative management of appendicitis in children
}

\author{
Helene Nepomuceno, Erik G. Pearson \\ Department of Pediatric Surgery, Sunrise Children's Hospital, Las Vegas, NV, USA \\ Contributions: (I) Conception and design: EG Pearson; (II) Administrative support: EG Pearson; (III) Provision of study materials or patients: None; \\ (IV) Collection and assembly of data: All authors; (V) Data analysis and interpretation: All authors; (VI) Manuscript writing: All authors; (VII) Final \\ approval of manuscript: All authors.
}

Correspondence to: Erik G. Pearson, MD, FACS. 653 N. Town Center, Blvd Suite \#412, Las Vegas, NV, USA. Email: erikgpearson@gmail.com.

\begin{abstract}
Appendicitis is a common condition in childhood and adolescence that frequently requires urgent surgical intervention. For almost two centuries appendicitis has been recognized as a medical problem with a surgical solution. Currently the appendix can be removed with a minimally invasive approach, low anesthetic and surgical risk, and swift hospital discharge. Despite these advances, surgery and anesthesia have associated risks including postoperative infection, bleeding, hernia and organ injury among others. In addition, surgery requires time off of school and work to recover and associated healthcare costs can be significant. In both adult and pediatric populations, quality data suggesting a nonoperative approach is suggesting a change to the traditional surgical paradigm. Adults studies have demonstrated both safety and efficacy in the nonoperative management of acute appendicitis. In selected children with uncomplicated appendicitis, initial nonoperative management has been shown to be safe with fewer complications, fewer disability days and less healthcare costs while avoiding the risks inherent to surgery. Ongoing randomized controlled clinical trials in both the United States and Europe seek to further demonstrate the safety of nonoperative management and assist physicians with educating patients about the risk profile of their treatment decision. In complicated appendicitis presenting with abscess or acute appendiceal phlegmon, an initial nonoperative strategy with or without abscess drainage followed by interval appendectomy is the current state of the art though the utility of interval appendectomy is questioned.
\end{abstract}

Keywords: Appendicitis; pediatric appendicitis; nonoperative appendicitis; appendectomy

Received: 28 April 2020; Accepted: 23 June 2020; Published: 25 July 2021.

doi: $10.21037 /$ tgh-20-191

View this article at: http://dx.doi.org/10.21037/tgh-20-191

\section{Background}

Appendicitis is the most common abdominal surgical emergency in children with a lifetime risk of $8 \%$ and median age of incidence between 10 and 11 years $(1,2)$. Acute appendicitis is a tremendous burden on the healthcare system as it accounts for over $11 \%$ of pediatric emergency room visits and over 70,000 hospital admissions annually $(3,4)$. The classical presentation of the disease includes the gradual onset of abdominal pain, initially periumbilical and then migrating to the right lower quadrant frequently accompanied by fever, loss of appetite and emesis. Appendicitis is known for its progressive nature as the disease evolves from uncomplicated inflammation at the onset of symptoms to perforation usually within 48 hours. The clinical spectrum of appendicitis in children extends from the child who is minimally symptomatic with focal right lower quadrant pain to one in septic shock complicated by perforation, intra-abdominal abscess, and disseminated infection. In young children (less than 5 years) the signs and symptoms of appendicitis may deviate from the classical presentation. Perforation is most common in young children who are less able to communicate symptoms, with rates reported of up to $100 \%$ in infants 1 year of age and $82 \%$ for children under 5 years (5).

The evolution of diagnosis has progressed quite rapidly in the last several decades with the development of scoring systems (Alvarado, Pediatric Appendicitis Score) and 
increased expertise in ultrasound to augment the clinical exam $(6,7)$. Resuscitation, antibiotics and surgery have been the mainstay of therapy for over the last century and the current state of the art is a simple, minimally invasive operation with few complications and a short hospital stay. In the last decade this approach has been challenged with the findings of safe and satisfactory outcomes with the nonoperative management of selected patients with acute uncomplicated appendicitis. In children with complicated appendicitis, presenting with intraabdominal abscess or phlegmon, initial nonoperative treatment with intravenous antibiotics and abscess drainage (if indicated) followed by interval appendectomy is a safe and acceptable strategy (8).

\section{Evolution of surgical management of appendicitis}

Prior to the well referenced description of "perforating inflammation of the vermiform appendix" by Dr. Reginald Fitz in 1886, surgeons had been extirpating the appendix and discovering the evolution of appendicitis as a clinical disease process (9). The first surgeon on record, Claudius Amyand, eponymous for Amyand's hernia (an appendix identified within a hernia sac) identified an inflamed appendix in an inguinal hernia of a young boy in 1736 (10). Amyand tied off and removed the appendix and the boy made a good recovery. Following Amyand, several descriptions of appendicitis as a cause of morbidity and death are found including John William Keys Parkinson in 1812 who presented an inflamed perforated appendix to the Medico-Chirurgical Society after removing it post mortem of a 5 year old boy who died from peritonitis resulting from the perforation (10).

The milestone in the surgical management of the disease came with McBurney's description of the surgical approach that bears his name in 1894 . McBurney's open operation included an oblique incision, approximately 4 inches in length that "crosses a line drawn from the anterior iliac spine to the umbilicus, nearly at right angles about one inch from the iliac spine, and is so situated that its upper third lies above that line." (11). McBurney evolved his surgical approach from his original vertical paramedian incision to get better exposure of the base of the appendix. Following McBurney, the appendectomy literature lay quiet for nearly a century until Semm, a German gynecologist and pioneer of minimally invasive surgery, published his paradigm-shifting paper on endoscopic appendectomy in 1983 (12). Following this publication the literature explodes with refinements in technique and comparisons of open to minimally invasive appendectomy. A decade after the first description of minimally invasive appendectomy came prospective, randomized clinical data demonstrating its superiority to open appendectomy $(\mathrm{OA})$ in terms of surgical site infection, hospital length of stay and pain (13). While the minimally invasive approach is the gold standard surgical approach for appendicitis when resources are abundant, OA remains the gold standard in resource poor areas though this continues to evolve (14).

Now we stand at the age of another paradigm shift in the clinical management of appendicitis. As our expertise in diagnostic imaging and antibiotic therapy has become more refined the utility of surgery in selected patients is questioned. Currently in most centers across the United States, children with acute appendicitis are treated initially with antibiotics and fluids once the diagnosis is made followed shortly by laparoscopic appendectomy. In some centers across the United States the management decisions have changed to nonoperative management in selected children and this has been demonstrated to be safe and effective with an acceptable risk profile.

\section{Introducing nonoperative treatment for acute appendicitis}

Appendectomy is a simple operative solution to the problem of appendicitis with low risk, a short hospital stay and quick recovery. The "low" risk profile of appendicitis still includes surgical site infection, incisional hernias, bleeding, organ injury, pain and risks associated with general anesthesia. Over 3\% of pediatric appendectomies performed at urban centers and $5 \%$ of appendectomies in rural hospitals are referred to as negative appendectomies when the pathological evaluation demonstrates a normal appendix (15). In these cases, the surgery was performed and the patient was exposed to risk unnecessarily. The cost of appendectomy is variable across the United States reaching $\$ 7,000$ to $\$ 8,000$ in both rural and urban hospitals and more if there are postoperative complications (15). For many diseases traditionally managed operatively, the safety, efficacy and cost savings found in nonoperative management spurred interest in applying this approach to appendicitis, a disease that affects up to $8 \%$ of Americans in their lifetime $(16,17)$.

Clinical evidence supports the nonoperative management of appendicitis across multiple trials and analyses in both adults and children. A 2012 meta-analysis of five randomized 
control trials demonstrated nonoperative management of acute uncomplicated appendicitis, without abscess or phlegmon, was associated with fewer complications, improved pain control, and less sick leave among adults (18). These benefits came at the expense of higher rates of recurrence with antibiotic management compared to appendectomy. In 2015, the Finnish Appendicitis Acuta (APPAC) noninferiority randomized clinical trial enrolled 530 adults aged $18-60$ years and demonstrated that most patients who received nonoperative treatment did not require appendectomy during the 1 year follow-up and those who did require surgery did not have an increased risk of complications (19). At 5-year follow-up, recurrence was 39\% in the nonoperative group (20). Complications occurred in $24 \%$ of patients receiving appendectomy compared to $7 \%$ in the nonoperative group and patients in the appendectomy group required 11 more days of sick leave than those patients treated with antibiotics alone. After 5-year follow-up the APPAC trial demonstrated that $61 \%$ of patients could safely avoid surgery and its associated complications and recovery time. In the Italian NOTA (Nonoperative Treatment for Acute Appendicitis) study, 159 patients with suspected appendicitis were enrolled and treated with amoxicillin and clavulanate with a two-year follow-up (21). In their population they found that nonoperative treatment had only a $14 \%$ recurrence rate at two years and the majority of these patients were successfully treated with a subsequent course of antibiotics and avoidance of surgery. While there is evidence supporting the utilization of a nonoperative management strategy in adults there is also evidence to proceed with caution. In a systematic review with meta-analysis of five randomized controlled trials including 1,351 patients, Podda and colleagues identified a significantly higher treatment efficacy (98\% vs. $76 \%$ ) at 1 year follow-up in patients treated with appendectomy compared with antibiotic therapy alone (22). In addition, in the patients treated nonoperatively, $23 \%$ developed recurrent appendicitis within 1 year and the rate of complicated appendicitis identified at the time of operation was higher in those patients failing nonoperative treatment $(20 \%$ vs. $9 \%)$.

In adults, one major concern with the adoption of nonoperative management of appendicitis is the risk of malignancy. Cancer is not insignificant in the pathological examination of adult appendix specimens. In an analysis of 3,554 adult appendectomy specimens there was a $0.8 \%$ overall rate of cancer including adenocarcinoma, low grade mucinous neoplasm and neuroendocrine tumors (23). In a separate study in Germany, 1,033 adult appendix specimens were reviewed with an overall appendiceal neoplasm rate of $2.8 \%$ (24). While these studies demonstrate a relatively low rate of neoplasm, it should still be included in the differential diagnosis, especially in adults with advanced age and a large appendiceal diameter. In children the rate of neoplasm is very low, approximately $0.3 \%$ and the majority of these tumors are appendiceal carcinoids (25).

There is substantial evidence to support nonoperative management of acute appendicitis in children. Nonoperative management of appendicitis is of particular interest to pediatric surgeons in order to avoid the known risks of general anesthesia and abdominal surgery, especially as negative appendectomy rates in children still approach greater than $6 \%$ in some centers (26). In order for the current operative paradigm to shift toward nonoperative management across all centers, urban and rural, specialized pediatric and general hospitals, treatment will need to be safe and efficacious, with low recurrence, minimal absence from school and suitable cost effectiveness.

\section{Outcomes of nonoperative treatment of appendicitis in children}

There is a growing body of literature supporting the nonoperative treatment of acute nonperforated appendicitis in pediatric patients. A mix of retrospective, prospective studies as well as recent and ongoing randomized control trials have demonstrated that nonoperative management is feasible, safe, and cost-effective in early, uncomplicated appendicitis. Data from a retrospective study between 2003-2006 examined 16 of 136 children managed nonoperatively with acute appendicitis suggested by clinical exam and ultrasound (27). They identified that 15 of 16 children (94\%) had resolution of symptoms and reduction in size of the appendix on US within 48 hours. A feasibility study between January and October 2011 compared operative to nonoperative management of early acute appendicitis in 24 patients, finding that in appropriately selected children nonoperative management is safe and effective (28). Between 2007-2013 a prospective study of nonoperative management of acute appendicitis asked parents to select operative or nonoperative treatment at admission (29). In the nonoperative arm they found 99\% initial success for nonoperative treatment; however, $29 \%$ of children suffered recurrence at 4.3 years followup. Most of these recurrences occurred in the first year with higher rates among patients with appendicolith. With respect to patient satisfaction between operative 
and nonoperative management Tanaka found that satisfaction levels were higher among patients who received laparoscopic appendectomy. In a recent prospective study between 2015 and 2018, 146 children ( $<16$ years) were initially treated nonoperatively for uncomplicated appendicitis with cutoffs of persistent pain, prolonged fever or increased white blood count at 12 hours (30). Ultimately nonoperative management failed in $51 \%$ of children and perforation was identified in $19 \%$ of these cases. They concluded that children younger than 7 years or with fever persisting longer than 1 day may benefit from initial surgery rather than nonoperative management. Across the non-randomized studies in children on nonoperative management, while antibiotics alone achieve a high rate of success in selected patients, recurrence of disease and need for surgery at some point within the first year following diagnosis is significant.

Data from retrospective and prospective studies set the stage for a randomized control trial to evaluate the safety and efficacy of nonoperative management in children with appendicitis. A randomized control trial performed in Sweden enrolled fifty patients with imaging-confirmed acute uncomplicated appendicitis (31). The success rate, defined as resolution of symptoms without significant complications, for nonoperative treatment was $92 \%$ with one child requiring appendectomy during the primary antibiotic period. By one year of follow-up, one child had recurrent appendicitis requiring appendectomy and 6 patients required appendectomy for recurrent pain (5) or parental wish (1) with overall $62 \%$ of children not requiring appendectomy. The team followed up the children at 5 years and an additional 2 patients developed recurrent acute appendicitis requiring appendectomy and ultimately $54 \%$ of children were able to avoid surgery by the end of follow-up (32). Importantly, contrary to adult data, there was no increase in the rate of complicated appendicitis if initial management was nonoperative.

Following this randomized control trial, a number of multicenter trials are currently in progress. A feasibility randomized control trial, the CONTRACT (CONServative Treatment of Appendicitis in Children) has been proposed in the United Kingdom (33). It aims to enroll patients aged 4-15 years and main outcomes will include recruitment rate (primary outcome), identification of strategies to optimize recruitment, performance, clinical outcomes, and safety. Two non-inferiority trials are underway. The APPY trial is a multicenter non-inferiority randomized control trial examining the primary treatment outcome of treatment failure, defined as recurrent appendicitis within 1 year or negative appendectomy, as well as secondary outcomes including complications, time to discharge, and the number and duration of hospital admissions during the first year (34). The APRES Trial out of Australia is a prospective noninferiority randomized control study that will measure the primary outcome of unplanned or unnecessary operation and complications at 30 days (35). Secondary outcomes will include length of stay, complications at 1 year, time of work and school, analgesic requirements, and cost. Another Amsterdam-based multicenter non-inferiority randomized control trial by the APAC group (Antibiotics versus Primary Appendectomy in Children) will randomize children across 15 hospitals (36). The primary outcome is rate of complications at 1 year, with a noninferiority margin of $5 \%$. Secondary outcomes will include delayed appendectomy, quality of life, pain, and cost. A multiinstitutional prospective patient choice trial for nonoperative management and surgery for uncomplicated pediatric appendicitis in the United States is underway and primary outcomes include success rate and differences in disability days, and secondary outcomes include complication rates, quality of life, and healthcare satisfaction (37). One question that becomes increasingly salient given a growing supply of quality data is patient and parent choice given two safe and efficacious options.

In a one-year prospective patient choice cohort study, Minneci and colleagues determined the effectiveness of nonoperative management of acute appendicitis with parents choosing between nonoperative management and appendectomy following surgical consultation (38). They included children aged 7-17 years with 48 hours or less of abdominal pain, a white blood cell count less than $18,000 / \mu \mathrm{L}$, radiographic evidence of nonruptured acute appendicitis with an appendiceal diameter of $1.1 \mathrm{~cm}$ or less without fecalith, abscess or phlegmon and a surgical consultation confirming a clinical diagnosis of acute appendicitis. Children were excluded from the study if they had diffuse peritonitis, imaging suspecting perforation, C-reactive protein levels higher than $40 \mathrm{mg} / \mathrm{L}$, a positive pregnancy test or a history of chronic abdominal pain. If parents chose the nonoperative arm of the study the child was admitted and started on intravenous piperacillin/tazobactam with oral food and liquids withheld for at least 12 hours. If they improved with decreasing pain or tenderness their diet was advanced and the child was given oral amoxicillin/ clavulanate and discharged with a 10-day total course. If the child had clinical worsening it was considered a failure and 
a prompt laparoscopic appendectomy was performed. Any child discharged who returned with abdominal pain and a clinical workup consistent with acute appendicitis underwent laparoscopic appendectomy. Children in the surgery arm of the study were admitted to the hospital, promptly started on intravenous antibiotics and received a laparoscopic appendectomy within 12 hours. Follow-up was completed up to 1 year for children in both arms of the study. Of the 629 patients who presented with acute appendicitis, 136 met inclusion criteria and 102 patients were enrolled with 37 choosing nonoperative management and 65 choosing surgery. The success rate of nonoperative management was $95 \%$ initially at hospital discharge declining to $76 \%$ at 21 months with 9 children requiring appendectomy. Minneci and colleagues identified that the incidence of complicated appendicitis was similar in the two groups, children in the nonoperative group who required appendectomy did not experience post-operative complications, and health related quality of life assessments were similar in children receiving appendectomy and antibiotics alone. Nonoperative management was associated with significantly fewer disability days at 1 year ( $8 v s .21$ days) and lower total appendicitisrelated health care costs at 1 year $(\$ 4,219$ vs. $\$ 5,029)$ compared to children receiving urgent appendectomy. This study helps clarify the safety and efficacy of managing selected children nonoperatively for acute uncomplicated appendicitis without fecalith and demonstrates the opportunity for significant cost savings and limited time away from school and work. In a follow-up study focusing on patient education and patient choice a patient activation tool (PAT) was developed to assist in the decision making between surgery or nonoperative management (39). While the authors found that the PAT did not improve measures of decision making, they identified that families are well informed and well prepared to participate in their child's treatment decision.

Acute appendicitis associated with appendicolith has demonstrated high risks of failure and an increased incidence of complicated appendicitis. Up to $10 \%$ of patients with appendicitis may present with appendicolith, which may lead to obstruction of the appendiceal lumen that cannot regress with antibiotic therapy alone. One meta-analysis reported an increased risk for nonoperative treatment failure, with $50 \%$ of patients with appendicolith undergoing antibiotic therapy requiring appendectomy (40). Nonoperative management of acute appendicitis with an appendicolith in children has an unacceptably high failure rate according to the available evidence.
Approximately $30 \%$ of children present with perforated appendicitis, with higher rates among younger children who are less communicative $(4,5)$. Several retrospective series exist of children treated nonoperatively with good outcomes in cases without abscess, phlegmon, or mass. A prospective randomized trial compared nonoperative treatment to early appendectomy in unselected children with perforated appendicitis without abscess or mass (41). The primary outcome of time away from normal activities was significantly reduced with early appendectomy and the overall adverse event rate was significantly lower for early appendectomy (30\%) versus interval appendectomy performed at 6-8 weeks (55\%). A cost analysis based on this trial showed significantly lower costs associated with early compared with interval appendectomy, related to the significant increase in adverse events in the interval appendectomy group (42).

In the case of associated periappendiceal abscess or phlegmon, an initial nonoperative management approach with antibiotics and/or abscess drainage is an effective strategy. A randomized controlled trial compared initial nonoperative management with drainage and interval appendectomy to initial laparoscopic appendectomy (43). There were no significant difference in total hospitalization, recurrent abscess, or overall charges, although initial appendectomy required a longer operative time. A 2019 meta-analysis examined the comparison of nonoperative management with delayed appendectomy versus early appendectomy for the treatment of complicated acute appendicitis (8). This was inclusive of two randomized control and 12 observational studies analyzing 1,288 patients both combined and as subsets. Pooled data demonstrated that nonoperative management alone had a $90 \%$ success rate and $15.4 \%$ recurrent appendicitis rate with complication and re-admission rates significantly lower for initial nonoperative management for abscess or phlegmon. If free perforation was found without abscess or phlegmon initial operative management is preferred. Length of stay was significantly shorter for operative management, and there was no significant difference in cost between treatments.

Interval appendectomy has traditionally been offered after successful nonoperative management of perforated appendicitis due to the perceived risk of recurrent appendicitis. In a survey performed in 2003 , over $80 \%$ of North American pediatric surgeons routinely offered interval appendectomy (44). A 2009 retrospective study found that recurrence rates in children with complicated 
appendicitis who were successfully managed nonoperatively was $8 \%$, a relatively low incidence that may be used to argue against routine performance of interval appendectomy (45).

\section{Conclusions}

Acute appendicitis is the most common surgical emergency in childhood and presents with a spectrum from uncomplicated to perforated with complications including abscess and phlegmon. Appendicitis has traditionally been managed with urgent appendectomy and this is done with a minimally invasive technique and low surgical and anesthetic risk. Nonoperative management of uncomplicated acute appendicitis has shown both safety and efficacy across multiple studies and has demonstrated significant cost savings and limited disability time without increasing morbidity. Appropriate patient selection for nonoperative treatment is important as the current data support nonoperative treatment only in patients with specific criteria including limited duration of symptoms and imaging confirmation of uncomplicated appendicitis without fecalith. Patient and family education as well as inclusion of the parent into the treatment decision is incredibly important when choosing a management strategy. A shift toward nonoperative management would represent a paradigm shift for pediatric appendicitis, which is managed almost exclusively with appendectomy in the setting of uncomplicated, early acute appendicitis. Multiple randomized control trials are ongoing to compare the effectiveness of antibiotics alone versus urgent appendectomy and data from these trials will assist the clinician with providing appropriate education to families and to set expectations. The current data suggest that an initial trial of nonoperative management is safe and efficacious, does not increase the risk of complicated appendicitis, and overall is associated with fewer surgical complications, fewer disability days, and lower healthcare costs.

The management of complicated acute appendicitis remains controversial. Treatment options include immediate appendectomy, nonoperative management with drainage of abscess if indicated and interval appendectomy. Current data support initial nonoperative treatment including intravenous antibiotics and drainage of intraabdominal abscess as needed. Currently, most surgeons recommend interval appendectomy following an episode of complicated appendicitis managed nonoperatively though this currently is debated.
Appendicitis is a common condition that consumes a significant amount of healthcare resources and causes morbidity among both pediatric and adult communities. Nonoperative treatment of appendicitis in selected patients with uncomplicated disease is supported by quality data demonstrating decreased complications, fewer sick days and less time off of work and school. There are multiple randomized clinical trials in progress that will help clinicians make the decision between surgery and nonoperative treatment including the education of patients and their families.

\section{Acknowledgments}

Funding: None.

\section{Footnote}

Provenance and Peer Review: This article was commissioned by the Guest Editors (Eduardo Perez, Samir Pandya, and Matthew S. Clifton) for the series "Current Topics in Pediatric General Surgery" published in Translational Gastroenterology and Hepatology. The article has undergone external peer review.

Conflicts of Interest: Both authors have completed the ICMJE uniform disclosure form (available at http://dx.doi. org/10.21037/tgh-20-191). The series "Current Topics in Pediatric General Surgery" was commissioned by the editorial office without any funding or sponsorship. The authors have no other conflicts of interest to declare.

Etbical Statement: The authors are accountable for all aspects of the work in ensuring that questions related to the accuracy or integrity of any part of the work are appropriately investigated and resolved.

Open Access Statement: This is an Open Access article distributed in accordance with the Creative Commons Attribution-NonCommercial-NoDerivs 4.0 International License (CC BY-NC-ND 4.0), which permits the noncommercial replication and distribution of the article with the strict proviso that no changes or edits are made and the original work is properly cited (including links to both the formal publication through the relevant DOI and the license). See: https://creativecommons.org/licenses/by-nc$\mathrm{nd} / 4.0 \%$. 


\section{References}

1. Rentea RM, St Peter SD. Pediatric Appendicitis. Surg Clin North Am 2017;97:93-112.

2. Addiss DG, Shaffer N, Fowler BS, et al. The epidemiology of appendicitis and appendectomy in the United States. Am J Epidemiol 1990;132:910-25.

3. Wier LM, Yu H, Owens PL, et al. Overview of Children in the Emergency Department, 2010: Statistical Brief \#157. Healthcare Cost and Utilization Project (HCUP) Statistical Briefs. Rockville (MD) 2006.

4. Barrett ML, Hines AL, Andrews RM. Trends in Rates of Perforated Appendix, 2001-2010: Statistical Brief \#159. Healthcare Cost and Utilization Project (HCUP) Statistical Briefs. Rockville (MD) 2006.

5. Morrow SE, Newman KD. Current management of appendicitis. Semin Pediatr Surg 2007;16:34-40.

6. Gonzalez DO, Lawrence AE, Cooper JN, et al. Can ultrasound reliably identify complicated appendicitis in children? J Surg Res 2018;229:76-81.

7. Kulik DM, Uleryk EM, Maguire JL. Does this child have appendicitis? A systematic review of clinical prediction rules for children with acute abdominal pain. J Clin Epidemiol 2013;66:95-104.

8. Fugazzola P, Coccolini F, Tomasoni M, et al. Early appendectomy vs. conservative management in complicated acute appendicitis in children: A meta-analysis. J Pediatr Surg 2019;54:2234-41.

9. Huang ZH, Chiu YC, Ho LL, et al. Acute appendicitis complicated with necrotizing fasciitis in a patient with adult-onset Still's disease: A case report. Medicine (Baltimore) 2018;97:e9794.

10. Cope Z. The Growth of Knowledge of Acute Abdominal Diseases 1800-1900. Proc R Soc Med 1964;57:129-34.

11. McBurney C. IV. The Incision Made in the Abdominal Wall in Cases of Appendicitis, with a Description of a New Method of Operating. Ann Surg 1894;20:38-43.

12. Semm K. Endoscopic appendectomy. Endoscopy 1983;15:59-64.

13. Attwood SE, Hill AD, Murphy PG, et al. A prospective randomized trial of laparoscopic versus open appendectomy. Surgery 1992;112:497-501.

14. GlobalSurg Collaborative. Laparoscopy in management of appendicitis in high-, middle-, and low-income countries: a multicenter, prospective, cohort study. Surg Endosc 2018;32:3450-66.

15. Tom CM, Friedlander S, Sakai-Bizmark R, et al. Outcomes and costs of pediatric appendectomies at rural hospitals. J
Pediatr Surg 2019;54:103-7.

16. Ricciardi R, Baxter NN, Read TE, et al. Is the decline in the surgical treatment for diverticulitis associated with an increase in complicated diverticulitis? Dis Colon Rectum 2009;52:1558-63.

17. Broderick-Villa G, Burchette RJ, Collins JC, et al. Hospitalization for acute diverticulitis does not mandate routine elective colectomy. Arch Surg 2005;140:576-81; discussion 581-3.

18. Mason RJ, Moazzez A, Sohn H, et al. Meta-analysis of randomized trials comparing antibiotic therapy with appendectomy for acute uncomplicated (no abscess or phlegmon) appendicitis. Surg Infect (Larchmt) 2012;13:74-84.

19. Salminen P, Paajanen H, Rautio T, et al. Antibiotic Therapy vs Appendectomy for Treatment of Uncomplicated Acute Appendicitis: The APPAC Randomized Clinical Trial. JAMA 2015;313:2340-8.

20. Salminen P, Tuominen R, Paajanen H, et al. Five-Year Follow-up of Antibiotic Therapy for Uncomplicated Acute Appendicitis in the APPAC Randomized Clinical Trial. JAMA 2018;320:1259-65.

21. Di Saverio S, Sibilio A, Giorgini E, et al. The NOTA Study (Non Operative Treatment for Acute Appendicitis): prospective study on the efficacy and safety of antibiotics (amoxicillin and clavulanic acid) for treating patients with right lower quadrant abdominal pain and long-term follow-up of conservatively treated suspected appendicitis. Ann Surg 2014;260:109-17.

22. Podda M, Cillara N, Di Saverio S, et al. Antibioticsfirst strategy for uncomplicated acute appendicitis in adults is associated with increased rates of peritonitis at surgery. A systematic review with meta-analysis of randomized controlled trials comparing appendectomy and non-operative management with antibiotics. Surgeon 2017;15:303-14.

23. Kunduz E, Bektasoglu HK, Unver N, et al. Analysis of Appendiceal Neoplasms on 3544 Appendectomy Specimens for Acute Appendicitis: Retrospective Cohort Study of a Single Institution. Med Sci Monit 2018;24:4421-6.

24. Brunner M, Lapins P, Langheinrich M, et al. Risk factors for appendiceal neoplasm and malignancy among patients with acute appendicitis. Int J Colorectal Dis 2020;35:157-63.

25. Ranaweera C, Brar A, Somers GR, et al. Management of pediatric appendiceal carcinoid: a single institution experience from 5000 appendectomies. Pediatr Surg Int 
2019;35:1427-30.

26. Oyetunji TA, Ong'uti SK, Bolorunduro OB, et al. Pediatric negative appendectomy rate: trend, predictors, and differentials. J Surg Res 2012;173:16-20.

27. Abeş M, Petik B, Kazil S. Nonoperative treatment of acute appendicitis in children. J Pediatr Surg 2007;42:1439-42.

28. Armstrong J, Merritt N, Jones S, et al. Non-operative management of early, acute appendicitis in children: is it safe and effective? J Pediatr Surg 2014;49:782-5.

29. Tanaka Y, Uchida H, Kawashima H, et al. Long-term outcomes of operative versus nonoperative treatment for uncomplicated appendicitis. J Pediatr Surg 2015;50:1893-7.

30. Ikegami M, Miyano G, Nojiri S, et al. Indications for Nonoperative Management of Uncomplicated Appendicitis in Children: A Prospective Analysis at a Single Institution. J Laparoendosc Adv Surg Tech A 2020;30:70-5.

31. Svensson JF, Patkova B, Almstrom M, et al. Nonoperative treatment with antibiotics versus surgery for acute nonperforated appendicitis in children: a pilot randomized controlled trial. Ann Surg 2015;261:67-71.

32. Patkova B, Svenningsson A, Almstrom M, et al. Nonoperative Treatment Versus Appendectomy for Acute Nonperforated Appendicitis in Children: Five-year Follow Up of a Randomized Controlled Pilot Trial. Ann Surg 2020;271:1030-5.

33. Hutchings N, Wood W, Reading I, et al. CONTRACT Study - CONservative TReatment of Appendicitis in Children (feasibility): study protocol for a randomised controlled Trial. Trials 2018;19:153.

34. Hall NJ, Eaton S, Abbo O, et al. Appendectomy versus non-operative treatment for acute uncomplicated appendicitis in children: study protocol for a multicentre, open-label, non-inferiority, randomised controlled trial. BMJ Paediatr Open 2017;1:bmjpo-2017-000028.

35. Xu J, Liu YC, Adams S, et al. Acute uncomplicated appendicitis study: rationale and protocol for a multicentre, prospective randomised controlled non-inferiority study to evaluate the safety and effectiveness of non-operative management in children with acute uncomplicated appendicitis. BMJ Open 2016;6:e013299.

doi: $10.21037 /$ tgh-20-191

Cite this article as: Nepomuceno H, Pearson EG. Nonoperative management of appendicitis in children. Transl Gastroenterol Hepatol 2021;6:47.
36. Knaapen M, van der Lee JH, Bakx R, et al. Initial nonoperative management of uncomplicated appendicitis in children: a protocol for a multicentre randomised controlled trial (APAC trial). BMJ Open 2017;7:e018145.

37. Minneci PC, Hade EM, Lawrence AE, et al. Multiinstitutional trial of non-operative management and surgery for uncomplicated appendicitis in children: Design and rationale. Contemp Clin Trials 2019;83:10-7.

38. Minneci PC, Mahida JB, Lodwick DL, et al. Effectiveness of Patient Choice in Nonoperative vs Surgical Management of Pediatric Uncomplicated Acute Appendicitis. JAMA Surg 2016;151:408-15.

39. Minneci PC, Cooper JN, Leonhart K, et al. Effects of a Patient Activation Tool on Decision Making Between Surgery and Nonoperative Management for Pediatric Appendicitis: A Randomized Clinical Trial. JAMA Netw Open 2019;2:e195009.

40. Huang L, Yin Y, Yang L, et al. Comparison of Antibiotic Therapy and Appendectomy for Acute Uncomplicated Appendicitis in Children: A Meta-analysis. JAMA Pediatr 2017;171:426-34.

41. Blakely ML, Williams R, Dassinger MS, et al. Early vs interval appendectomy for children with perforated appendicitis. Arch Surg 2011;146:660-5.

42. Myers AL, Williams RF, Giles K, et al. Hospital cost analysis of a prospective, randomized trial of early vs interval appendectomy for perforated appendicitis in children. J Am Coll Surg 2012;214:427-34; discussion 434-5.

43. St Peter SD, Aguayo P, Fraser JD, et al. Initial laparoscopic appendectomy versus initial nonoperative management and interval appendectomy for perforated appendicitis with abscess: a prospective, randomized trial. J Pediatr Surg 2010;45:236-40.

44. Chen C, Botelho C, Cooper A, et al. Current practice patterns in the treatment of perforated appendicitis in children. J Am Coll Surg 2003;196:212-21.

45. Puapong D, Lee SL, Haigh P, et al. Routine interval appendectomy in children is not indicated. J Pediatr Surg 2007;42:1500-3. 